\title{
STUDENT PAPER: GPU Acceleration for SQL Queries on Large-Scale Distributed Systems
}

\author{
Linh Nguyen \\ Hampden-Sydney College \\ 1 College Road \\ Hampden-Sydney, VA 23943 USA \\ nguyenl16@hsc.edu
}

\author{
Paul Hemler \\ Hampden-Sydney College \\ 1 College Road \\ Hampden-Sydney, VA 23943 USA \\ phemler@hsc.edu
}

\begin{abstract}
General purpose GPUs are a powerful hardware with a number of applications in the realm of relational databases. We further extended a database framework designed to allow for GPU execution queries. Our technique is novel in that it implements Dynamic Parallelism, a new feature in recent hardware, to accelerate SQL JOINs. Query execution results in $1.25 \mathrm{X}$ speedup on average with respect to a previous method, also accelerated by GPUs, which employs a multi-dimensional CUDA Grid.

More importantly, we divided the queries to run on multiple BW nodes to investigate the scalability of both SELECT and JOIN.
\end{abstract}

\section{Keywords}

GPGPU, BWSIP, CUDA, SQL, Distributed Computing

\section{INTRODUCTION}

A relational database management system (RDBMS) manages the organization, storage, access, security, and integrity of data. Manipulating an RDBMS requires the use of Structured Query Language (SQL), an industry-standard declarative language capable of performing very complex queries and aggregations over data sets. In an RDBMS, these data sets are organized in structured tables, and SQL serves as an intermediary between a client program and its databases. The explosive growth of various types of unstructured data, has called for the next wave of innovation in data storage, management, and analysis, otherwise known as Big Data.

MapReduce presents one such innovation. Initially developed by Google, MapReduce is a programming paradigm widely used for data intensive and large-scale data analysis applications [10]. The architecture is simple abstraction that allows programmers to write a map function that processes key-value pair associated with the input data. The reduce function then merges all the intermediate values associated with the same intermediate key. The programming model involves three phases: Map; Shuffle and Sort; Reduce.

Permission to make digital or hard copies of all or part of this work for personal or classroom use is granted without fee provided that copies are not made or distributed for profit or commercial advantage and that copies bear this notice and the full citation on the first page. To copy otherwise, or republish, to post on servers or to redistribute to lists, requires prior specific permission and/or a fee. Copyright @JOCSE, a supported publication of the Shodor Education Foundation Inc.

DOI: https://doi.org/10.22369/issn.2153-4136/8/1/5
In some cases, a MapReduce framework has replaced traditional SQL database, though the advantage of one over another remains a debated topic. Both approaches are very general methods through which data can be processed. In fact, there are efforts to combine the two in order to ease programmers' learning curve. In particular, Apache Hive allows programmers to write queries in SQL-like fashion and then converts the queries to map/reduce [1]. Regardless of the specifics, SQL remains highly applicable in the Big Data era thanks to the power of its declarative syntax. As such, significant efforts have been made in improving the performance of SQL, targeting parallel heterogeneous hardware architectures, which incorporates many processor types in a single machine $[5,6,7,8,11,12,13,14,16]$.

The SQL model of data organization fits a parallel execution model extremely well since different processors can work on different portions of a table simultaneously. Among relevant attempts, the most promising approach currently utilizes the newest generation of Graphics Processing Units (GPUs), which are implemented as massively parallel architectures designed for rapidly rendering complex and realistic graphical scenes. More importantly, several general purpose software development frameworks for programming GPUs have become standard. Two such frameworks are Compute Unified Device Architecture (CUDA) [19] and the Open Compute Language (OpenCL) [17]. These frameworks allow applications to simply and effectively harness the power of the GPU. In addition, they have also become a common method of accelerating many compute-intensive applications $[9]$.

This project aims to extend a GPU-based database to allow for the execution of SQL queries on multiple GPUs contained within the Blue Waters (BW) supercomputer system [18]. The specific focus of this project targets two common but extremely important SQL queries: SELECT and JOIN. While most previous papers utilize parallel primitives, our implementation executes with an opcode virtual machine model. In short, the key contributions of this paper include:

- Dynamic Parallelism Approach - Earlier GPU hardware did not include the ability to launch new GPU functions directly from threads. The BW compute nodes provide newer GPUs with the Kepler Architecture, which includes this important feature, referred to as Dynamic Parallelism (DP). We employ DP to enhance hardware utilization in accelerating JOIN.

- Multi-GPU Configuration - To the best of our knowledge, all previous work has shown speedups on a single- 
GPU system. However, databases often reside on many compute nodes and querying data incurs additional expensive communication costs. Since the BW is a large-scale distributed system with thousands of nodes, it is particularly suitable to investigate this necessary aspect of both SELECT and JOIN queries.

- Educational Values - Work on this project has exposed me to a multitude of areas in Computational Sciences, such as Compilers, Computer Architecture, and Parallel Computing. Most importantly, I have gained significant experiences in multi-GPU programming. Our specific implementation is under the MapReduce dwarf. The relevant characteristics will be discussed in subsequent sections.

In summary, we performed an in-depth investigation of dividing queries across multiple GPUs and the implications of novel hardware features to explore the potential benefits of GPU acceleration for SQL queries.

\section{RELATED BACKGROUND}

This section provides overviews of SQL queries, and MapReduce. It also discusses current solutions in parallel SQL execution on the GPUs

\subsection{SQL SELECTs and JOINs}

In the following discussion, we denote a table as $T_{i}$ and each column in a table as $c_{i}$ where $i$ is their corresponding enumeration.

A SELECT statement extracts data from a database in an organized and readable format. A typical SELECT statement is accompanied by clauses such as FROM, WHERE, and ORDER BY. These clauses specify the conditions on which the data is filtered. A predicate includes one or more of these conditions.

$$
\begin{aligned}
& T_{1} \\
& \begin{array}{|c|c|}
\hline c_{1} & c_{2} \\
\hline 1 & \mathrm{w} \\
\hline 2 & \mathrm{z} \\
\hline 3 & \mathrm{z} \\
\hline
\end{array} \Rightarrow \begin{array}{|c|c|}
\hline c_{1} & c_{2} \\
\hline 2 & \mathrm{z} \\
\hline 3 & \mathrm{z} \\
\hline
\end{array}
\end{aligned}
$$

Figure 1: The result rows of a SELECT query: SELECT $*$ FROM $T_{1}$ WHERE $T_{1} . c_{1} \geq 2$

A JOIN query requires at least two tables that share a common attribute, referred to as a foreign key. While there are many types of JOINs, we focus only on the cross-join, denoted $\bowtie$, since it is the simplest JOIN query but still embodies all the computational characteristics of other JOINS. A cross-join computes the Cartesian product of all the keys in the relevant tables. Figure 2 highlights the result rows of a cross-join between $T_{1}$ and $T_{2}$ based on the predicate $T_{1} . c_{2}=T_{2} \cdot c_{2}$, where $c_{2}$ is the foreign key. While the result consists of nine rows in total, the predicate reduces the result to just three rows.

A SELECT query involves a loop that examines every row in a table. A JOIN entails a nested loop, where for each row in the first table, the corresponding loop iterates over the entire second table and emits rows that match the predicate. Clearly, this entire process is computationally demanding for very large tables with millions of rows.

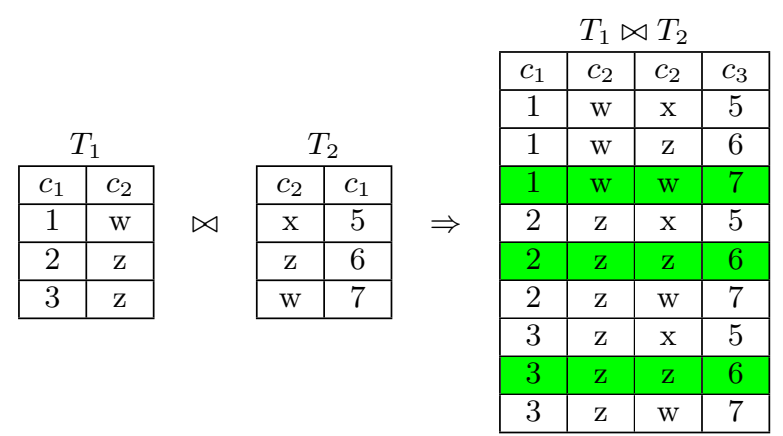

Figure 2: The cross join $T_{1} \bowtie T_{2}$ :

SELECT $*$ FROM $T_{1}, T_{2}$ WHERE $T_{1} \cdot c_{2}=T_{2} \cdot c_{2}$

\subsection{MapReduce}

As previously mentioned, the MapReduce paradigm is an active research area. The three main phases are shown in Figure 3.

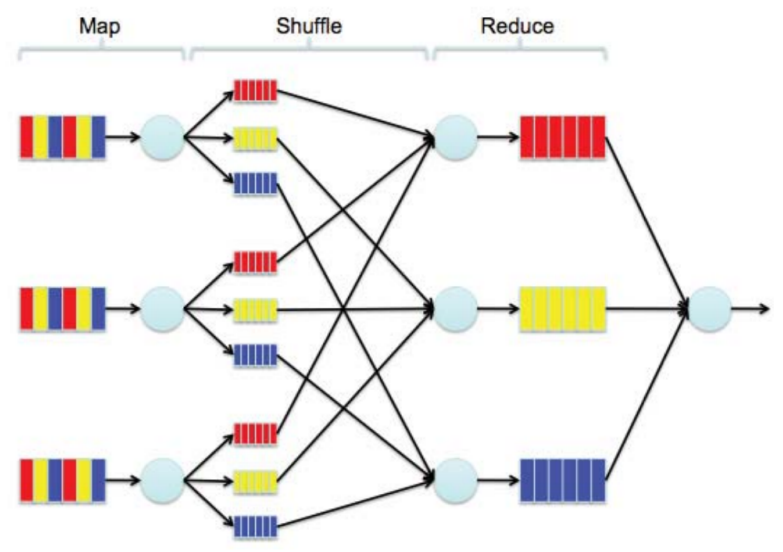

Figure 3: The three phases of MapReduce.

Map Phase: The input data is partitioned into splits and each is assigned to Map tasks to be processed in separate nodes in the cluster. These Map tasks perform computations on each input key-value pair from its assigned partition of data. Finally, they generate a set of intermediate results for each key.

Shuffle and Sort Phase: This phase sorts the data generated by the Map tasks from other nodes and divides this data into regions to be further processed by the Reduce task. Therefore, all Map tasks must complete prior to this phase. In addition, this phase distributes the data as necessary to nodes where the Reduce tasks will execute.

Reduce Phase: The Reduce tasks perform additional operations on the intermediate data by merging values associated with a particular key to a smaller set of values to produce the output. The number of Reduce tasks does not need to be equal to the number of Map tasks. For more complex data processing procedures, multiple MapReduce calls may be linked together in sequence.

MapReduce is a simple and scalable approach to achieve high speed and efficient parallel processing over a massive amount of data.

\subsection{Parallel SQL Databases}


There have been other works in the literature $[7,8,11,14]$ that employ the parallel primitives such as scan, sort, or filter to process queries on the GPUs. Each of these primitives is a kernel launched on a given set of data in an order specified by programmers. Every kernel then needs to retrieve relevant data from global memory. Fundamentally, this approach entails many memory accesses and consequently affects performance.

We build directly on the work presented in $[3,4,5,6]$. This line of research proposes using a virtual machine (VM) instead. From a high-level point of view, a VM is a sequence of jump instructions inside the GPU. Each instruction is identified by an opcode and performs a certain operation. Each thread is mapped to a data point, small enough to be loaded directly into a register.

The source code of the Virginian database, which embodies this approach, is open source [4]. While inspired by SQLite [2] , the initial implementation presents a completely custom VM, which we will describe in Section 3. We modified this VM to accommodate the distributed architecture of the BW system.

\section{IMPLEMENTATION}

The program first builds an abstract syntax tree (AST) from the SQL query. The AST is then processed in several passes to generate a virtual program, namely a set of jump instructions that represents the query. The details of the parsing algorithm and code generations are documented in the Virginian database page [4].

\subsection{Virtual Machine Infrastructure}

A VM is implemented as a CUDA kernel that executes a sequence of instructions procedurally. Each instruction is identified by an opcode and contains four registers. Each opcode serves a certain functionality.

The aforementioned work partitions a SQL table into chunks of data, called the tablet $[4,5]$. Each tablet is a fix-sized group of rows. As a result, accessing an entire table of data may involve multiple tablets, but accessing a single row of data involves only a single tablet. Partitioning is beneficial at two levels of parallelism, especially for SQL SELECT. First, for a heavily distributed table, each of this table's tablets resides on a different node and thus can be processed independently. Second, for each tablet, threads in a CUDA grid handle their corresponding rows.

Since SELECT operates only on single source table, an extension was developed to this model to support operations over two or more tables, namely JOIN [3]. CUDA's organization of threads into a grid of up to three dimensions coincides with the structure of a Cartesian product where a two-table join corresponds to a two dimensional grid. We recall the example from Figure 2, where two tables $T_{1}$ and $T_{2}$ are cross-joined on the same foreign key. Here, the $\mathrm{x}-$ dimension of the CUDA grid corresponds to the row index of $T_{1}$ while the y-dimension corresponds to row index of $T_{2}$. The shortcoming, however, is that this approach is limited to joining at most three tables since this is the maximum supported dimensions of a CUDA grid.

This method, which we will now refer to as the Grid method, is used as the baseline performance for our novel technique for accelerating JOIN, which will be discussed shortly. In addition, we scale both SELECT and JOIN to ex- ecute on multiple nodes and compare the performance with that of a single node.

\subsection{Dynamic Parallelism for SQL JOINs}

DP is a new functionality provided by the Kepler Architecture [19]. DP allows kernels to be launched from the device without going back to the host. The kernel, block or thread that initiates the launch is referred to as parent. Also, the kernel, block, or thread that is launched is referred to as the child. DP allows explicit synchronization between the parent and the child through a built-in device function. Launches can be nested from parent to child, then child to grandchild and so on. The deepest nesting level that requires explicit synchronization is the synchronization depth. Parent and child kernels have coherent access to global memory. Shared and local memories are exclusive for parent and child kernels.

The JOIN algorithm involves at least two data arrays, taken from the related data tables. Each thread takes one element from the first array in the JOIN predicate and finds the matching keys from the other array. DP implementation launches a kernel to gather the result elements in parallel for each thread.

\subsection{Multi-GPU Configuration}

To further improve the performance of large-scale data processing, it is essential to share workloads among distributed compute nodes equipped with GPUs. As discussed in Section 1, this project is the first to examine the possibility of breaking up a data set and running a query concurrently on multiple GPUs. Our custom data structure is useful in the context of a heavily distributed database by enabling efficient management of data between networked machines. In our implementation, tablets play a major role since they vastly simplify the process of moving data to and from different nodes. Tablets allow each node to operate exclusively on known-size records.

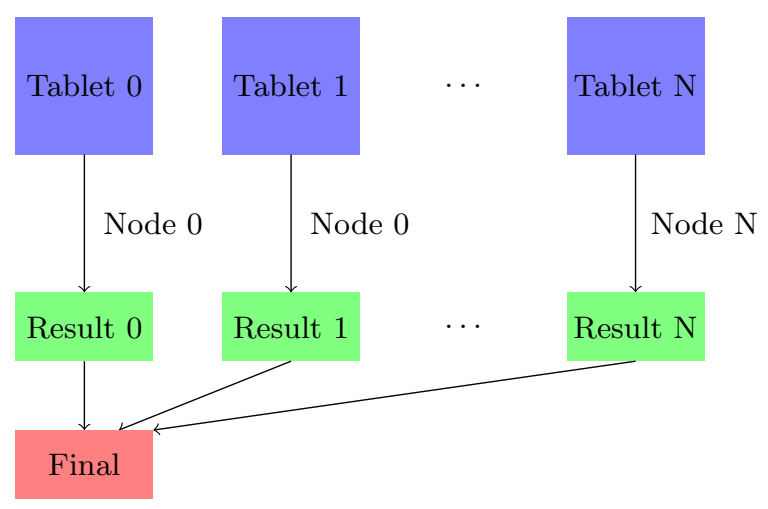

Figure 4: Query plan for SQL SELECT

A table is first divided into tablets. This step is trivial thanks to the partitioning scheme of a SQL table. Each tablet is sent to a XK compute node through MPI. On each node, each tablet is then processed by the same virtual machine row by row on the node's GPU. After producing its corresponding set of result rows, each node passes these rows back to the master node, which then reorganizes these rows and emits the final table to the user. Figure 4 illustrates this approach for the SELECT queries. 
$T_{1}$

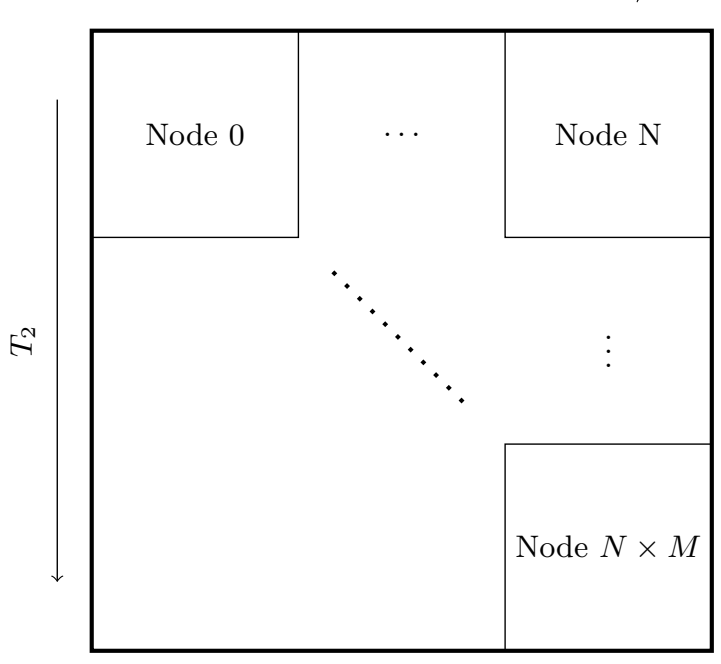

Figure 5: Query plan for SQL JOIN

Similarly, the two tables in a JOIN query are each divided into an array of tablets. Figure 5 demonstrates this plan. Table $T_{1}$ contains $\mathrm{N}$ tablets, and table $T_{2}$ contains M tablets. Since a JOIN statement is a Cartesian product of two arrays of data, each node is responsible for a different section of the cross product. For instance, Node 0 computes $T_{1}$.Tablet_0 $\bowtie T_{2}$.Tablet_0. The results of these computations are again passed to the master node in the same procedure as in Figure 4.

Splitting data across multiple nodes has a two-fold advantage: data can be processed more quickly and a larger quantity of data can be processed. The database can now support much larger tables while retaining the same performance. For the same data set, more computing powers reduce the processing time.

\section{RESULTS}

We adopted the test data from $[4,3,6]$, which includes 8 million rows randomly generated numerical values. The columns consists of an integer primary key and 2 columns each with distribution in $[-100,100]$, a normal distribution with standard deviation 5 , and another with standard deviation 20. Each of these distributions was generated once each for a 32-bit integer column and a IEEE 754 32-bit floating point column. The GNU Scientific Library was used to ensure the quality of the random distribution.

A suite of ten different queries were written to thoroughly test the different characteristics for both SELECT and JOIN. Hence, there are twenty queries in total. For SELECT, five of the queries for each suite involve integer values; the rest of the suite test floating point values. For JOIN, only one query operates exclusively on integer values; one query tests floating point exclusively; the rest of the suite test a arithmetic and conditional statements with a mixture of both integer and floating points.

We have little reason to believe results would change significantly with realistic data sets, since all rows are checked. Also, both textual and non-textual data are ultimately represented by numeric values. Besides, we have tested virtu- ally all basic possible combinations of common case queries.

This section also highlights the educational values of our project.

\subsection{Performance}

We analyze in detail the runtime growth of our implementation and then discuss performance improvement relatively to the baseline as well as the scalability of our implementation as a function of the number of nodes.

\subsubsection{SELECT}

The characteristics of runtime growth on a single node has been discussed [5]. We therefore focus mainly on the scaling factor of the implementation. Figure 6 visually presents the speedups observed as we scale our program. Single-node execution was predictably slower by factors proportional to the number of nodes used in computation.

\section{Scaling Performance of SELECT and JOIN}

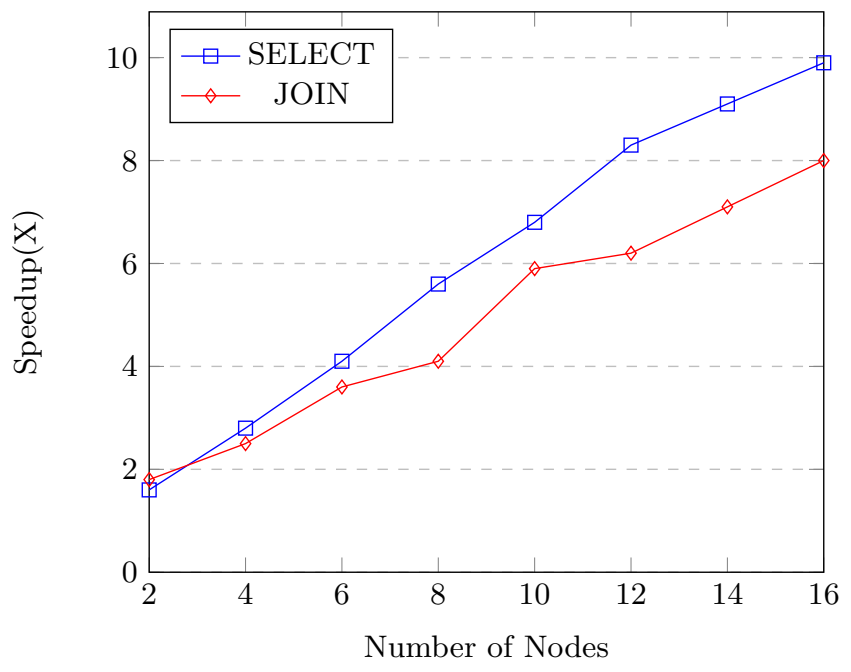

Figure 6: Scaling factors for SELECT and JOIN

\subsubsection{JOIN}

Test were performed using two 3500 row tables containing randomly generated values in the same layout as for SELECT. We chose this number of rows since the Cartesian product of two tables with 3500 rows each will space $3500 \cdot 3500=12,250,000$ rows, larger than the test number of rows for SELECT.

Figure 8(a) graphically demonstrates the differences in running times using DP and Grid methods. The mean execution time for all ten queries for Grid is 0.2526 seconds and 0.233 seconds for DP. We noticed that floating points are slightly faster and reported the average between integer and floating points. Figure 8(b) depicts the speedups of DP with respect to Grid, which averaged to be $1.245 \mathrm{X}$.

Scaling SQL JOINs on distributed is less straightforward than with SELECT. Since the Cartesian product is a twodimensional product, we chose the sizes of the two tables in Figure 5 as $\{1 \times 2,2 \times 2,2 \times 3,2 \times 4,3 \times 4,2 \times 7,4 \times 4\}$. These numbers correspond to the same number of nodes for SELECT and their speedups are also displayed in Figure 6. We chose these dimensions to examine the effects of the ra- 
Proportions of Rows Returned vs. Execution Time

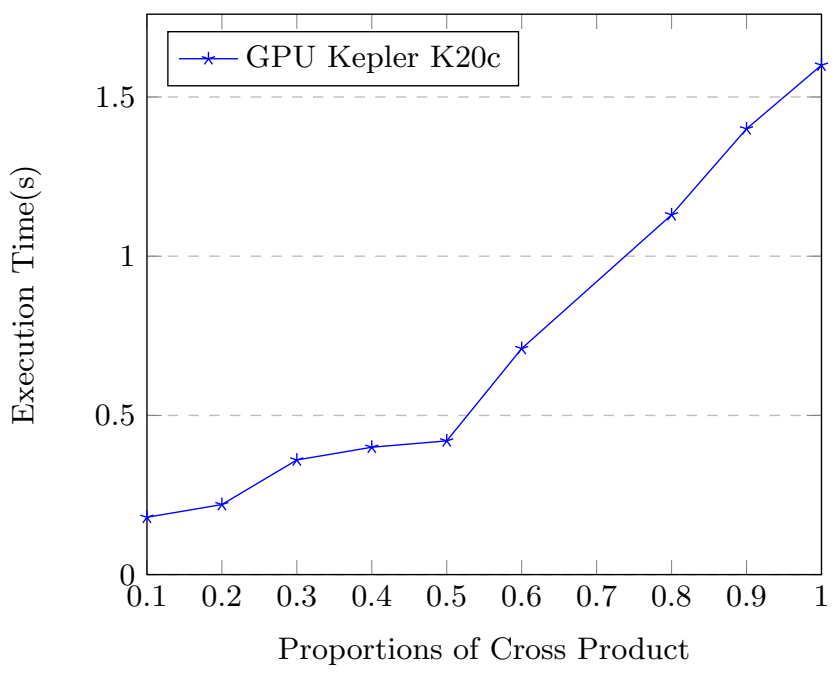

Figure 7: As fewer rows in the Cartesian product are filtered, the GPU becomes less efficient

tio $\frac{N}{M}$ have on the scalability of JOINs. The graph showed a weaker scalability for JOINs due to a more complex distribution plan. The mean percentage to theoretical speedup is $54.4 \%$.

Figure 9 provides the latency breakdown as percentages of critical parts in the execution cycle of a program. Predictably, as the number of nodes increases, the system incurs much more overhead to organize data to the assigned nodes. We notice a gradual decline in the role actual computation plays with respect to total runtime. In contrast, the nodeto-node communication overhead increases linearly, up to $75.2 \%$ at 16 nodes.

\subsection{Educational Values}

The use of GPU in HPC is becoming extremely popular due to the high computational power and high bandwidth coupled with the availability of (de facto) frameworks. However, effectively programming a hybrid system with MPI and CUDA is especially difficult as the growing complexities of applications require more and more processors. Because the communication patterns and resource scheduling are common to a wide range of domains, we generalize our experiences and hope that they could be helpful to future programmers faced with similar problems.

\subsubsection{Multi-GPU Programming}

MPI and CUDA combine the two parallel programming frameworks enables solving problems with a data size too large to fit into the memory of a single GPU, or that would require an unreasonably long compute time on a single node. Also, this combination allows programmers to efficiently accelerate existing MPI applications with GPUs.

In multi-GPU programming, an MPI launcher starts multiple instances of an application and distributes these instances across the nodes of the BW. Each instance then launches its own CUDA kernel. A major problem arises when each node does not receive an even amount of data or does not return the same amount of data, referred to (a) Query Execution Times

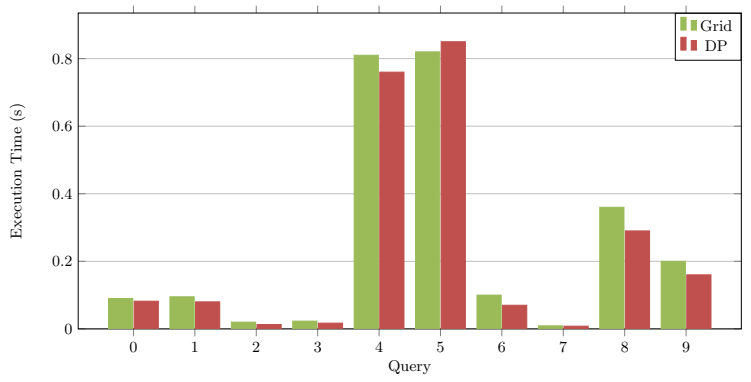

(b) DP Query Speedup

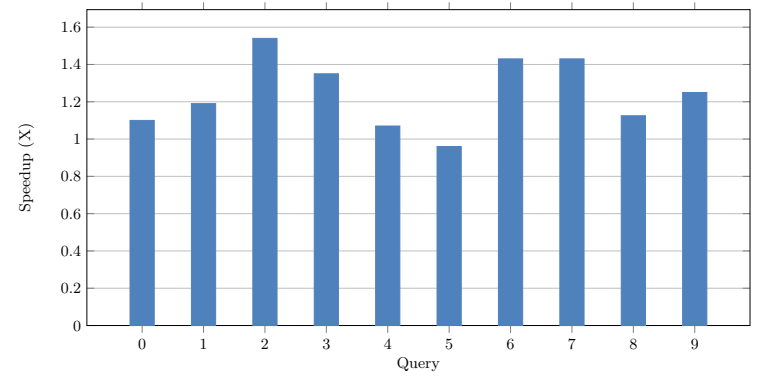

Figure 8: Variations of two different methods for accelerating JOINs

as workload imbalance. Our implementation automatically solves the first problem since data is evenly divided into tablets. The latter is harder to address since imbalance may occur due to the specific characteristics of an application. For instance, we chose a uniform distribution for our test data since the distribution has equal probability for any random region of data, thus minimizes the chance for load imbalance. It is critical for the programmer to be aware of these challenges in order to address them properly.

\subsubsection{MapReduce Introduction}

MapReduce has been shown to work well on the BW [15], which gives a compelling reason to develop a section on how to effectively utilize this paradigm. Database aggregation falls into the MapReduce dwarf since each stage is directly mapped to a phase in MapReduce, as described in Figure 3. In our case, the input data is the original tables and the output data is the result table.

Map: Each of the partitioned tablets in a table is mapped to a node. The node's number and its corresponding tablet form a key/value pair. Each node then executes the VM generated and then produces its own set of result rows. These are the intermediate results to be passed to next phase.

Shuffle and Sort: Each node sorts its own result table based on programmer's specification. For instance, these nodes arrange the numeric values in ascending/descending order.

Reduce: The master node is responsible for this phase, collecting other nodes' intermediate tables based on their keys and then rearrange these tables to into one single final table to display.

\section{CONCLUSION}

The Virginian database was used as a platform for the 


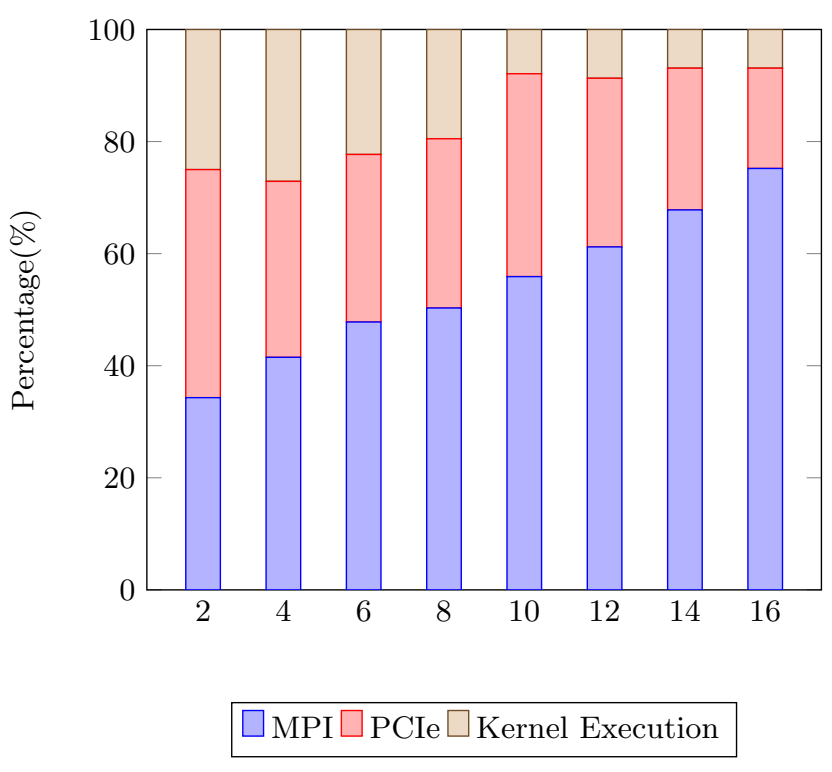

Figure 9: The fraction of each query runtime due to core parts of computation

project, enabling the use of an existing SQL parsing mechanism and switching between host and device. Execution on the GPU was supported by a completely custom VM implemented as a CUDA kernel. Our DP approach shows an average of $1.245 \mathrm{X}$ faster than the previously implemented Grid method. The characteristics of each query, the type of data being queried, the size of the result set, and the numbers of nodes involved were all significant factors in the performance of GPU-enabled database. Despite these variation, the minimum speedup for DP was 1.1X.

SQL is an excellent interface through which the GPU can be accessed: it is much simpler and more widely used than many alternatives. Using SQL represents a break from the paradigm of previous research which drove GPU queries through the use of operational primitives. Additionally, SQL dramatically reduces the effort required to employ GPUs for database acceleration. While still enjoying the simplicity of SQL, users can now benefit from a much reduced runtime. All the details are hidden so that users do not need to learn any additional materials besides their existing SQL knowledge.

Our opcode model allows the programmer to choose any granularity for database operation in conjunction with a relatively simple VM, while also enabling efficient data handling. Programmers can simply add or modify opcodes to support more complex queries. We also generalize the module to help with the learning curve by presenting the general challenges associated with multi-GPU programming as well as the project's relation to MapReduce.

\section{REFLECTIONS}

The Blue Waters Student Internship Program (BWISP) impacts me significantly on many aspects of my personal and professional developments.

The Petascale Institute (PI) allowed me the opportunity to learn how to use the Blue Waters supercomputer, using the various parallel programming frameworks it pro- vides. This experience and exchanges with my fellow students and the instructors improved my programming skills greatly. Also, the exposure to other disciplines though these conversations opened my eyes to the vast application of Computer Science (CS). The areas I was introduced to include biomathematics, physical simulations, and financial modelling.

After the PI, working on my own project helped me apply these learned skills in practice. I gained invaluable experiences working with large software project that utilizes many software tools and involves various areas of CS, such as Compilers, Computer Architecture, and Software Development. More importantly, the experience provides me with the materials to discuss in my graduate school applications. As a result, I was accepted to all programs I applied to, including well-known institutions in High Performance Computing such as University of Michigan and Georgia Tech.

All in all, the BWISP was an immense success and equipped me with the necessary skills to succeed in my career as a Computer Science researcher.

\section{FUTURE WORK}

Our implementation has been designed partly to demonstrate a very general framework for GPU data processing. Using this framework, a next step is to implement and test additional database features such as other types of join, including inner,outer, and natural Joins. Modern RDBMSs are extremely complex, and much more work in this area is required to fully replicate this functionality in a GPUfriendly manner. We have shown that our opcode framework will be adaptable to this additional functionality, with modifications to our VM as appropriate to facilitate inter-opcode communication $[4,3]$.

Regardless of the direction, the general area of GPU application development is ripe for future research.

\section{ACKNOWLEDGMENTS}

This work is supported by a grant from the Shodor Education Foundataion through the Blue Waters Student Internship Program (BWSIP) and by the National Center for Supercomputing Applications, who provides the hardware used in this project.

\section{REFERENCES}

[1] apache hive. https://hive.apache.org/. Date accessed: 2016-04-11.

[2] the sqlite virtual machine. https://www.sqlite.org/opcode.html. Date accessed: 2016-04-06.

[3] K. Angstadt and E. Harcourt. A virtual machine model for accelerating relational database joins using a general purpose gpu. In Proceedings of the Symposium on High Performance Computing, HPC '15, pages 127-134, San Diego, CA, USA, 2015. Society for Computer Simulation International.

[4] P. Bakkum. The Virginian Database. https://github.com/bakks/virginian. Date accessed: 2015-05-10.

[5] P. Bakkum and S. Chakradhar. Efficient data management for gpu databases. Technical report, NEC Laboratories America, Princeton, NJ, 2013. 
[6] P. Bakkum and K. Skadron. Accelerating sql Database Operations on a GPU with CUDA. In Proceedings of the 3rd Workshop on General-Purpose Computation on Graphics Processing Units (GPGPU' 10), pages 94-103, New York, NY, 2010. ACM.

[7] S. Baxter. Relational Joins. https://nvlabs.github.io/moderngpu/join.html. Date accessed: 2016-03-19.

[8] S. Bress, M. Heimel, N. Siegmund, L. Bellatreche, and G. Saake. Gpu-accelerated database systems: Survey and open challenges. 2014.

[9] S. Che, J. W. Sheaffer, M. Boyer, L. G. Szafaryn, L. Wang, and K. Skadron. A characterization of the rodinia benchmark suite with comparison to contemporary cmp workloads. In Workload Characterization (IISWC), 2010 IEEE International Symposium on, pages 1-11, Dec 2010.

[10] J. Dean and S. Ghemawat. Mapreduce: Simplified data processing on large clusters. Commun. ACM, 51(1):107-113, Jan. 2008.

[11] N. K. Govindaraju, B. Lloyd, W. Wang, M. Lin, and D. Manocha. Fast computation of database operations using graphics processors. In Proceedings of the 2004 ACM SIGMOD International Conference on Management of Data, SIGMOD '04, pages 215-226, New York, NY, USA, 2004. ACM.

[12] R. J. Halstead, I. Absalyamov, W. A. Najjar, and V. J. Tsotras. Fpga-based multithreading for in-memory hash joins. In Conference on Innovative Data System Research (CIDR' 15), 2015.

[13] R. J. Halstead, B. Sukhwani, H. Min, M. Thoennes, P. Dube, S. Asaad, and B. Iyer. Accelerating join operation for relational databases with fpgas. In Field-Programmable Custom Computing Machines (FCCM), 2013 IEEE 21st Annual International Symposium on, pages 17-20, April 2013.

[14] B. He, M. Lu, K. Yang, R. Fang, N. K. Govindaraju, Q. Luo, and P. V. Sander. Relational query coprocesing on graphics processors. ACM Trans. Database Syst., 34(4):21:1-21:39, Dec. 2009.

[15] G. B. W. K. Manisha Gajbe, Kalyana Chadalavada. Benchmarking and performance studies of mapreduce / hadoop framework on blue waters supercomputer. In WorldComp 15 - ABDA'15 International Conference on Advances in Big Data Analytics. ISBN, 2015.

[16] S. Meki and Y. Kambayashi. Acceleration of relational database operations on vector processors. Systems and Computers in Japan, 31(8):79-88, 2000.

[17] Advanced Micro Devices. Opencl programming guide. http://developer.amd.com/wordpress/media/2013/07/ AMD_Accelerated_Parallel_Processing_OpenCL_ Programming_Guide-rev-2.7.pdf. Date accessed: 2015-05-10.

[18] National Center for Supercomputing Applications. Brief blue waters system overview. https://bluewaters.ncsa.illinois.edu/user-guide. Date accessed: 2016-03-19.

[19] NVIDIA Corporation. Nvidia cuda programming guide. http://docs.nvidia.com/cuda/pdf/CUDA_C_ Programming_Guide.pdf. Date accessed: 2015-05-10. 